\title{
Cardiac Atrial Pacing Malfunction
}

National Cancer Institute

\section{Source}

National Cancer Institute. Cardiac Atrial Pacing Malfunction. NCI Thesaurus. Code C99921.

The cardiac rhythm device malfunction affected atrial pacing. (ACC) 